\title{
Customer Impatience in Multiserver Queues
}

\author{
Pallabi Medhi ${ }^{1}$, Amit Choudhury ${ }^{2}$ \\ ${ }^{1}$ Department of Statistics, Cotton College State University, Guwahati-781001, Assam, India \\ ${ }^{2}$ Department of Statistics, Gauhati University, Guwahati-781014, Assam, India
}

\begin{abstract}
In the modeling of many queuing systems, it is assumed that customers who arrive stay on till they receive service. In real life, this does not always happen. Arriving customer may decide against joining the system. In queuing parlance, this is known as balking. In this paper, we shall assume that customers may balk if service is not instantly available. Even if a customer joins the system, the customer may withdraw and leave without completely receiving service. This is known as reneging. In this paper, we consider a multiserver Markovian queuing system where customers may balk as well as renege. In addition to the traditional performance measures, some freshly designed ones have also been presented. The relevance of this work stems from the fact that not withstanding related analysis of similar customer behavior already available in literature, explicit closed form expressions are still not available for $M / M / k$ model. In this paper, we present the same. A numerical problem with design aspects has also been presented to demonstrate results derived.
\end{abstract}

Keywords: Balking, Impatience, Queuing, Reneging

2000 Mathematics Subject Classification: 60K25, 68M20, 90B22

\section{Introduction}

Queuing is a 'phenomenon that we all encounter as part of our everyday lives. In fact, one is almost certain to encounter some form of queue, or waiting line, in every walking hour' (Ravindran et.al, 1987). These days' customers are very demanding. Very often, they are very hard pressed for time. Consequently, long waiting times are frowned upon. Customers get impatient on the prospect of waiting. Such impatience translates into two types of customer behavior. First, if on its arrival, a customer finds the queuing system non-empty, it might decide not to join the queue. In queuing parlance, this is known as balking. Haight (1957) has provided a rationale which might influence a person to balk. It relates to the perception of the importance of being served which induces an opinion somewhere in between urgency, so that a queue of certain length will not be joined, to indifference where a non-zero queue is also joined. Even if the customer joins the queue, it might not be willing to wait for a long period in order to complete receiving service. The phenomena where a customer having joined the queuing system leaves it before completing service is known as reneging. Even though queuing models of various specifications have been discussed and analyzed in literature, it is not very often that the aspects of reneging and balking have been dealt with. This paper is an attempt in this direction.

We assume that balking is state dependent. Such an assumption implies that higher the queue size, higher is the probability that a customer may balk. It is not difficult to find many queuing systems where such customer behavior can be observed.

Reneging can be of two types- reneging till beginning of service (henceforth referred to as R_BOS) and reneging till end of service (henceforth referred to as R_EOS). R_BOS can be observed in queuing systems where a customer can renege only as long as queue. Once it begins receiving service, it cannot renege. A common example is the barbershop. A customer can renege while he is waiting in queue. However once service gets started i.e. hair cut begins, the customer cannot leave till hair cutting is over. On the other hand, R_EOS can be observed in queuing systems where a customer can renege not only while waiting in queue but also while receiving service. An example is processing or merchandising of perishable goods.

In this paper, we analyze a multi server Markovian queuing system $\mathrm{M} / \mathrm{M} / \mathrm{k}$ under the assumption that customers may balk as well renege. Two types of reneging R_BOS and R_EOS are discussed separately. To the best of our knowledge, a focused analysis generating explicit closed form expressions has not been carried out for this model with both types of reneging and state dependent balking. The arrival and service rates are assumed as $\lambda$ and $\mu$ respectively. We assume that each arriving customer has probability $\left(1-\mathrm{p}^{\mathrm{n}}\right.$ ${ }^{\mathrm{k}+1}$ ) of balking from a system with no idle servers where ' $n$ ' is the state of the system and ' $\mathrm{k}$ ' is the number of servers. As for reneging, each customer joining the system is assumed to have random patience time following $\exp (v)$.

The subsequent sections of this paper are structured as follows. Section 2 contains a brief review of the literature. Section 3 and section 4 contains the derivation of steady state probabilities and performance measures respectively. We perform sensitivity analysis in section 5. A numerical example is discussed in section 6. Concluding statements are present in section 7 . The appendix presented in section 8 contains some derivation.

\section{Literature Survey}

One of the earliest work on reneging was by Barrer (1957) where he considered deterministic reneging with single server markovian arrival and service rates. Customers were selected randomly for service. In his subsequent work, Barrer (1958) also considered deterministic reneging (of both R_BOS and R_EOS type) in a multiserver scenario with FCFS discipline. The general method of solution was 


\section{International Journal of Science and Research (IJSR) \\ ISSN (Online): 2319-7064}

Index Copernicus Value (2015): 78.96 | Impact Factor (2015): 6.391

extended to two related queuing problems. Another early work was by Haight (1959). Ancher and Gafarian (1963) carried out an early work on markovian reneging with markovian arrival and service pattern. Ghosal (1963) considered a $\mathrm{D} / \mathrm{G} / 1$ model with deterministic reneging. Gavish and Schweitzer (1977) also considered a deterministic reneging model with the additional assumption that arrivals can be labeled by their service requirement before joining the queue and arriving customers are admitted only if their waiting plus service time do not exceed some fixed amount. This assumption is met in communication systems. Kok and Tijms (1985) considered a single server queuing system where a customer becomes a lost customer when its service has not begun within a fixed time. Haghighi et al (1986) considered a Markovian multiserver queuing model with balking as well as reneging. Each customer had a balking probability which was independent of the state of the system. Reneging discipline considered by them was R_BOS. Liu et al (1987) considered an infinite server Markovian queuing system with reneging of type R_BOS. Customers had a choice of individual service or batch service, batch service being preferred by the customer. Brandt et al (1998) considered a S-server system with two FCFS queues, where the arrival rates at the queues and the service may depend on number of customers ' $n$ ' being in service or in the first queue, but the service rate was assumed to be constant for $\mathrm{n}>\mathrm{s}$. Customers in the first queue were assumed impatient customers with deterministic reneging. Boots and Tijms (1999) considered an M/M/C queue in which a customer leaves the system when its service has not begun within a fixed interval after its arrival. In this paper, they have given the probabilistic proof of 'loss probability', which was expressed in a simple formula involving the waiting time probabilities in the standard $\mathrm{M} / \mathrm{M} / \mathrm{C}$ queue. Wang et al (1999) considered the machine repair problem in which failed machines balk with probability (1-b) and renege according to a negative exponential distribution. Another work using the concepts of balking and reneging in machine interference queue has been carried out by Al-Seedy and Al-Ibraheem (2001). Bae et al. (2001) considered a M/G/1 queue with deterministic reneging. They derived the complete formula of the limiting distribution of the virtual waiting time explicitly. Choi et al. (2001) introduced a simple approach for the analysis of the $\mathrm{M} / \mathrm{M} / \mathrm{C}$ queue with a single class of customers and constant patience time by finding simple Markov process. Applying this approach, they analyzed the $\mathrm{M} / \mathrm{M} / 1$ queue with two classes of customer in which class 1 customer have impatience of constant duration and class 2 customers have no impatience and lower priority than class 1 customers. Performance measures of both $\mathrm{M} / \mathrm{M} / \mathrm{C}$ and $\mathrm{M} / \mathrm{M} / 1$ queues were discussed. Zhang et al. (2005) considered a M/M/1/N framework with Markovian reneging where they derived the steady state probabilities and formulated a cost model. Some performance measures were also discussed. A numerical example was discussed to demonstrate how the various parameters of the cost model influence the optimal service rates of the system. Choudhury (2008) analyzed a single server Markovian queuing system with the added complexity of customers who are prone to giving up whenever its waiting time is larger than a random thresholdhis patience time. He assumed that these individual patience times were independent and identically distributed exponential random variables. Reneging till beginning of service was considered. A detailed and lucid derivation of the distribution of virtual waiting time in the system was presented. Some performance measures were also presented. El- Paoumy (2008) also derived the analytical solution of $\mathrm{M}^{\mathrm{x}} / \mathrm{M} / 2 / \mathrm{N}$ queue for batch arrival system with Markovian reneging. In this paper, the steady state probabilities and some performance measures of effectiveness were derived in explicit forms. Another paper on Markovian reneging was by Yechiali and Altman (2008). They derived the probability generating function of number of customers present in the system and some performance measures were discussed. Choudhury (2009) considered a single server finite buffer queuing system $(\mathrm{M} / \mathrm{M} / 1 / \mathrm{K})$ assuming reneging customers. Both rules of reneging were considered and various performance measures presented under both rules of reneging.

Other attempts at modeling reneging phenomenon include those by Baccelli et al (1984), Martin and Artalejo (1995), Shawky (1997), Choi, Kim and Zhu (2004), and Singh et al (2007), El- Sherbiny (2008) and El-Paoumy and Ismail (2009) etc.

An early work on balking was by Haight (1957). Haghighi et al (1986) considered a Markovian multiserver queuing model with balking as well as reneging. Each customer had a balking probability which was independent of the state of the system. Reneging discipline was considered as R_BOS. Liu et al (1987) considered an infinite server Markovian queuing system with reneging of type R_BOS. Customers had a choice of individual service or batch service: batch service being preferred by the customer. Brandt et al (1998) considered a S-server system with two FCFS queues, where the arrival rates at the queues and the service may depend on number of customers ' $n$ ' being in service or in the first queue, but the service rate was assumed to be constant for $\mathrm{n}>\mathrm{s}$. Customers in the first queue were assumed impatient customers with deterministic reneging. Wang et al (1999) considered the machine repair problem in which failed machines balk with probability (1-b) and renege according to a negative exponential distribution. Another work using the concepts of balking and reneging in machine interference queue has been carried out by Al-Seedy and Al-Ibraheem (2001).

There have been some papers in which both balking as well as reneging were considered. Here mention may be made of the work by Haghighi et al (1986), Zhang et al (2005), ElPaoumy (2008), El- Sherbiny (2008), Shawky and ElPaoumy (2009).

\section{The System State Probabilities}

In this section, the steady state probabilities are derived by the Markov process method. We first analyze the case where customers renege only from the queue. Under R_BOS, let $\mathrm{p}_{\mathrm{n}}$ denote the probability that there are ' $n$ ' customers in the system. The steady state probabilities under R_BOS are

$$
\begin{gathered}
\lambda p_{0}=\mu p_{1} \\
\lambda p_{n-1}+(n+1) \mu p_{n+1}=\lambda p_{n}+n \mu p_{n} ; \mathrm{n}=1,2, \ldots \mathrm{k}-1
\end{gathered}
$$

\section{Volume 6 Issue 1, January 2017




\section{International Journal of Science and Research (IJSR)}

ISSN (Online): 2319-7064

Index Copernicus Value (2015): 78.96 | Impact Factor (2015): 6.391

$$
\lambda p^{n-k} p_{n-1}+\{k \mu+(n-k+1) v\} p_{n+1}=\lambda p^{n-k+1} p_{n}+\{k \mu+(n-k) v\} p_{n} ; \mathrm{n}=\mathrm{k}+1, \ldots \ldots
$$

Solving recursively, we get (under R BOS)

$$
\begin{gathered}
p_{n}=\left\{\lambda^{n} /\left(n ! \mu^{n}\right)\right\} p_{0} ; \mathrm{n}=1,2, \ldots \mathrm{k} \quad \text { (3.4) } \sum_{n=0}^{\infty} p_{n}=1 \text { and is given as } \\
p_{n}=\left[\lambda^{n} p^{\{(n-k)(n-k+1)\} / 2} /\left\{k ! \mu^{k} \prod_{r=k+1}^{n}(k \mu+\overline{r-k} v)\right\}\right] p_{0} \\
; \mathrm{n}=\mathrm{k}+1, \ldots \\
p_{0}=\left[\sum_{n=0}^{k} \lambda^{n} /\left(n ! \mu^{n}\right)+\sum_{n=k+1}^{\infty} \lambda^{n} p^{\{(n-k)(n-k+1)\} / 2} /\left\{k ! \mu^{k} \prod_{r=k+1}^{n}(k \mu+\overline{r-k} v)\right\}\right]^{-1} .
\end{gathered}
$$

where $\mathrm{p}_{0}$ is obtained from the normalizing condition
The steady state probabilities satisfy the recurrence relation. Under R BOS

$p_{n}=\{\bar{\lambda} /(n \mu)\} p_{n-1} ; \mathrm{n}=1,2, \ldots \mathrm{k}$,

and $p_{n}=\left\{\lambda p^{(n-k)} /(k \mu+\overline{n-k} v)\right\} p_{n-1} ; \mathrm{n}=\mathrm{k}+1$,

$\mathrm{k}+2, \ldots \ldots$

We shall denote by $K_{R_{-}}$BOS the probability that an arriving unit has to wait on arrival (under R_BOS). Then

$\mathrm{K}_{\mathrm{R} \_ \text {BOS }}=\operatorname{Pr}(\mathrm{N} \geq \mathrm{k})$

$$
=\sum_{n=k}^{\infty} p_{n} .
$$

We may call $K_{R_{\_} \text {Bos }}$ as Erlang's second (Erlang's delay probability) formula for balking and reneging (R_BOS) in line with similar nomenclature in Medhi (2003, page 87).

Under R_EOS where customer may renege from the queue as well as while receiving service, let $\mathrm{q}_{\mathrm{n}}$ denote the probability that there are $\mathrm{n}$ customers in the system.
Applying the Markov theory, we obtain the following set of steady state equations.

$$
\begin{gathered}
\lambda q_{0}=(\mu+v) q_{1}, \\
\lambda q_{n-1}+(n+1)(\mu+v) q_{n+1}=\lambda q_{n}+n(\mu+v) q_{n} ;
\end{gathered}
$$$$
\mathrm{n}=1,2, \ldots \mathrm{k}-1
$$

$$
\lambda p^{n-k} q_{n-1}+\{k \mu+(n+1) v\} q_{n+1}=\lambda p^{n-k+1} q_{n}+\{k \mu+n v\} q_{n}
$$

Solving recursively, we get (under R_EOS)

$$
\begin{array}{r}
q_{n}=\left[\lambda^{n} /\left\{n !(\mu+v)^{n}\right\}\right] q_{0} ; \mathrm{n}=1,2, \ldots . \mathrm{k}(3.11) \\
q_{n}=\left[\lambda^{n} p^{\{(n-k)(n-k+1)\} / 2} /\left\{k !(\mu+v)^{k} \prod_{r=k+1}^{n}(k \mu+r v)\right\}\right] q_{0}
\end{array}
$$$$
; \mathrm{n}=\mathrm{k}+1, \ldots(3.12)
$$

where $\mathrm{q}_{0}$ is obtained from the normalizing condition $\sum_{n=0}^{\infty} q_{n}=1$ and is given as

$$
q_{0}=\left[\sum_{n=0}^{k} \lambda^{n} /\left\{n !(\mu+v)^{n}\right\}+\sum_{n=k+1}^{\infty} \lambda^{n} p^{\{(n-k)(n-k+1)\} / 2} /\left\{k !(\mu+v)^{k} \prod_{r=k+1}^{n}(k \mu+r v)\right\}\right]^{-1}
$$

The recurrence relations under R_EOS are

$$
q_{n}=[\lambda /\{n(\mu+v)\}] q_{n-1} ; \quad \mathrm{n}=1,2, \ldots \mathrm{k},
$$

and $q_{n}=\left\{\lambda p^{(n-k)} /(k \mu+n v)\right\} q_{n-1} ; \mathrm{n}=\mathrm{k}+1, \mathrm{k}+2, \ldots \ldots$

We shall denote by $K_{R_{\text {EEOS }}}$ the probability that an arriving unit has to wait on arrival (under R_EOS). Then $\mathrm{K}_{\mathrm{R}_{\_} \text {EOS }}=\operatorname{Pr}(\mathrm{N} \geq \mathrm{k})$

$$
=\sum_{n=k}^{\infty} q_{n} .
$$

which may be called Erlang's second (Erlang's delay probability) formula for balking and reneging (R_EOS).

\section{Performance Measures}

An important measure is ' $\mathrm{L}$ ' which denotes the mean number of customers in the system. To obtain an expression for the same, we note that $\mathrm{L}=\mathrm{P}^{\prime}(1)$ where

$P^{\prime}(1)=\left.\frac{d}{d s} P(s)\right|_{s=1}$.

Here $\mathrm{P}(\mathrm{S})$ is the p.g.f. of the steady state probabilities. The derivation of $\mathrm{P}^{\prime}(1)$ is given in the appendix. From (8.1.8) and (8.2.4) the mean system size under two reneging rules are

$$
L_{R_{-} B O S}=(1 / v)\left[\lambda\left(1-K_{R_{-} B O S}\right)+\left\{\left(\lambda p_{0} K_{R_{-} B O S}(p \lambda, \mu, v)\right) / p^{k-1} p_{0}(p \lambda, \mu, v)\right\}-(\mu-v) \sum_{n=1}^{k} n p_{n}-k\left(K_{R_{-} B O S}-p_{k}\right)(\mu-v)\right](4.1)
$$

where $p_{0}(p \lambda, \mu, v)$ and $K_{R_{-} B O S}(p \lambda, \mu, v)$ are given in (8.1.5) and (8.1.7) respectively, and

$$
L_{R_{-} E O S}=(1 / v)\left[\lambda\left(1-K_{R_{-} E O S}\right)+\left\{\left(\lambda q_{0} K_{R_{-} E O S}(p \lambda, \mu, v)\right) / p^{k-1} q_{0}(p \lambda, \mu, v)\right\}-\mu \sum_{n=1}^{k} n q_{n}-k \mu\left(K_{R_{-} E O S}-q_{k}\right)\right]
$$




\section{International Journal of Science and Research (IJSR) \\ ISSN (Online): 2319-7064 \\ Index Copernicus Value (2015): 78.96 | Impact Factor (2015): 6.391}

where $q_{0}(p \lambda, \mu v)$ and $K_{R_{E} E S}(p \lambda, \mu \nu)$ are given in

(8.2.4) and (8.2.5) respectively.

Mean queue size can now be obtained and are given by

$$
\begin{aligned}
L_{q\left(R_{-} B O S\right)} & =L_{R_{-} B O S}-\sum_{n=1}^{k} n p_{n}-k\left(K_{R_{-} B O S}-p_{k}\right) \\
& =(1 / v)\left[\lambda\left(1-K_{R_{-} B O S}\right)+\left\{\left(\lambda p_{0} K_{R_{-} B O S}(p \lambda, \mu, v)\right) / p^{k-1} p_{0}(p \lambda, \mu, v)\right\}-\mu \sum_{n=1}^{k} n p_{n}-k \mu\left(K_{R_{-} B O S}-p_{k}\right)\right] . \\
L_{q\left(R_{-} E O S\right)} & =L_{R_{-} E O S}-\sum_{n=1}^{k} n q_{n}-k\left(K_{R_{-} E O S}-q_{k}\right) \\
& =(1 / v)\left[\lambda\left(1-K_{R_{-} E O S}\right)+\left\{\left(\lambda q_{0} K_{R_{-} B O S}(p \lambda, \mu, v)\right) / p^{k-1} q_{0}(p \lambda, \mu, v)\right\}-(\mu+v) \sum_{n=1}^{k} n q_{n}-k(\mu+v)\left(K_{R_{-} E O S}-q_{k}\right)\right] .
\end{aligned}
$$

Using Little's formula, one can calculate the average waiting time in the system and average waiting time in queue from the above mean lengths both under R_BOS and R_EOS.

Customers arrive into the system at the rate $\lambda$. However all the customers who arrive do not join the system because of balking. The effective arrival rate into the system is thus different from the overall arrival rate and is given by

$$
\begin{gathered}
\lambda_{\left(R_{-} B O S\right)}=\lambda \sum_{n=0}^{k-1} p_{n}+\lambda \sum_{n=k}^{\infty} p^{n-k+1} p_{n} \\
=\lambda\left(1-K_{R_{-} B O S}\right)+\left\{\lambda p_{0} K_{R_{-} B O S}(p \lambda, \mu, v)\right\} / \\
\left\{p^{k-1} p_{0}(p \lambda, \mu, v)\right\} .
\end{gathered}
$$$$
\operatorname{Avgrr}_{\left(R_{-} \mathrm{BOS}\right)}=\sum_{n=k+1}^{\infty}(n-k) v p_{n}
$$$$
=v\left\{L_{R_{-} B O S}-\sum_{n=1}^{k} n p_{n}\right\}-v k\left\{1-\sum_{n=0}^{k} p_{n}\right\}
$$$$
=\lambda\left(1-K_{R_{-} B O S}\right)+\left\{\lambda p_{0} K_{R_{-} B O S}(p \lambda, \mu, v)\right\} /\left\{p^{k-1} p_{0}(p \lambda, \mu, v)\right\}-\mu \sum_{n=1}^{k} n p_{n}-k \mu\left(K_{R_{-} B O S}-p_{k}\right) \text {. }
$$

$$
\begin{aligned}
& \text { Similarly in case of R_EOS } \\
& \begin{array}{c}
\lambda^{e}{ }_{\left(R_{-} E O S\right)}=\lambda\left(1-K_{R_{-} E O S}\right)+\left\{\lambda q_{0} K_{R_{-} E O S}(p \lambda, \mu, v)\right\} / \\
\left\{p^{k-1} q_{0}(p \lambda, \mu, v)\right\} .
\end{array}
\end{aligned}
$$

We have assumed that each customer has a random patience time following $\exp (v)$. Clearly then, the reneging rate of the system would depend on the state of the system as well as the reneging rule. The average reneging rate (avg rr) under the two reneging rules are given by

$$
\begin{aligned}
A v g r r_{\left(R_{-} E O S\right)} & =\sum_{n=1}^{\infty} n v q_{n} \\
& =v L_{R_{-} E O S} \\
& =\lambda\left(1-K_{R_{-} E O S}\right)+\left\{\lambda q_{0} K_{R_{-} E O S}(p \lambda, \mu, v)\right\} /\left\{p^{k-1} q_{0}(p \lambda, \mu, v)\right\}-\mu \sum_{n=1}^{k} n q_{n}-k \mu\left(K_{R_{-} E O S}-q_{k}\right) .
\end{aligned}
$$

In a real life situation, customers who balk or renege represent the business lost. Customers are lost to the system in two ways, due to balking and due to reneging. Management would like to know the proportion of total customers lost in order to have an idea of total business lost.

Hence the mean rate at which customers are lost (under R_BOS) is

$$
\begin{aligned}
& \lambda-\lambda^{e}{ }_{\left(R_{-} B O S\right)}+\operatorname{avgrr}_{\left(R_{-} B O S\right)}, \\
& =\lambda-\mu \sum_{n=1}^{k} n p_{n}-k \mu\left(K_{R_{-} B O S}-p_{k}\right) .
\end{aligned}
$$

and the mean rate at which customers are lost (under R_EOS) is

$$
\begin{aligned}
& \lambda-\lambda^{e}{ }_{\left(R_{-} E O S\right)}+\operatorname{avgrr} r_{\left.R_{-} E O S\right)}, \\
& =\lambda-\mu \sum_{n=1}^{k} n q_{n}-k \mu\left(K_{R_{-} E O S}-q_{k}\right) .
\end{aligned}
$$




\section{International Journal of Science and Research (IJSR) \\ ISSN (Online): 2319-7064 \\ Index Copernicus Value (2015): 78.96 | Impact Factor (2015): 6.391}

These rates helps in the determination of proportion of customers lost which is of interest to the system manager as also an important measure of business lost. This proportion (under R_BOS) is given by

$$
\begin{aligned}
& \left\{\lambda-\lambda^{e}{\left(R_{-} B O S\right)}+\operatorname{avgrr}_{\left(R_{-} B O S\right)}\right\} / \lambda, \\
& =1-(1 / \lambda)\left[\mu \sum_{n=1}^{k} n p_{n}+k \mu\left(K_{R_{-} B O S}-p_{k}\right)\right] .
\end{aligned}
$$

and the proportion (under R_EOS) is given by

$$
\begin{aligned}
& \left\{\lambda-\lambda^{e}{\left(R_{-} E O S\right)} \operatorname{avgrr}_{\left(R_{-} E O S\right)}\right\} / \lambda, \\
& =1-(1 / \lambda)\left[\mu \sum_{n=1}^{k} n q_{n}+k \mu\left(K_{R_{-} E O S}-q_{k}\right)\right] .
\end{aligned}
$$

The proportion of customers completing receipt of service can now be easily determined from the above proportion. The customers who leave the system from the queue do not receive service. Consequently, only those customers who reach the service station constitute the actual load of the server. From the server's point of view, this provides a measure of the amount of work he has to do. Let us call the rate at which customers reach the service station as $\lambda^{\mathrm{s}}$.Then under R BOS

$\lambda^{\mathrm{s}}{ }_{\left(\mathrm{R}_{-} \mathrm{BOS}\right)}=\lambda^{\mathrm{e}}{ }_{\left(\mathrm{R}_{-} \mathrm{BOS}\right)}$ (1-proportion of customers lost due to reneging out of those joining the system)

$$
\begin{aligned}
& =\lambda^{e}{ }_{\left(R_{-} B O S\right)}\left\{1-\sum_{n=k+1}^{\infty}(n-k) v p_{n} / \lambda^{e}{\left(R_{-} B O S\right)}^{\infty}\right\} \\
& =\lambda^{e}{\left(R_{-} B O S\right)}-a v g r r_{\left.R_{-} B O S\right)} \\
& =\mu \sum_{n=1}^{k} n p_{n}+k \mu\left(K_{R_{-} B O S}-p_{k}\right) .
\end{aligned}
$$

In case of R_EOS, one needs to recall that customers may renege even while being served and only those customers who renege from the queue will not constitute any work for the server. Then $\lambda^{\mathrm{s}}{ }_{\text {(R_EOS) }}=\lambda^{\mathrm{e}}$ (R_EOS) (1-proportion of customers lost due to reneging from the queue out of those joining the system)

$$
=\lambda_{\left(R_{-} E O S\right)}^{e}\left\{1-\sum_{n=k+1}^{\infty}(n-k) v q_{n} / \lambda^{e}{ }_{\left(R_{-} E O S\right)}\right\}
$$

$$
\begin{aligned}
& =\lambda^{e}{\left(R_{-} E O S\right)}-v\left\{Q^{\prime}(1)-\sum_{n=1}^{n} n q_{n}\right\}+k v\left(1-\sum_{n=0}^{k} q_{n}\right) \\
& =\lambda^{e}{\left(R_{-} E O S\right)}-v Q^{\prime}(1)+v \sum_{n=1}^{n} n q_{n}+k v\left(K_{R_{-} E O S}-q_{k}\right) \\
& =(\mu+v) \sum_{n=1}^{k} n q_{n}+k(\mu+v)\left(K_{R_{-} E O S}-q_{k}\right) .
\end{aligned}
$$

In order to ensure that the system is in steady state it is necessary for the rate of customers reaching the service station to be less than the system capacity. This translates to $\left(\lambda^{s} / k \mu\right)<1$.

\section{Sensitivity Analysis}

It is interesting to examine and understand how server utilization varies in response to change in system parameters. The three system parameters of interest are $\lambda, \mu, v$. We place below the effect of change in these system parameters on server utilization. For this purpose, we shall follow the following convention in the rest of this section.

$\mathrm{p}_{\mathrm{n}}(\lambda, \mu, v)$ and $\mathrm{q}_{\mathrm{n}}(\lambda, \mu, v)$ will denote the probability that there are ' $\mathrm{n}$ ' customers in a system with parameters $\lambda, \mu, v$ in steady state under R_BOS and R_EOS respectively.

i) If $\lambda_{1}>\lambda_{0}$, then

$\frac{p_{0}\left(\lambda_{1}, \mu, v\right)}{p_{0}\left(\lambda_{0}, \mu, v\right)}<1$

$\Rightarrow \frac{\left(\lambda_{0}-\lambda_{1}\right)}{\mu}+\ldots+\frac{\left(\lambda_{0}{ }^{k}-\lambda_{1}{ }^{k}\right)}{k ! \mu^{k}}+\frac{p\left(\lambda_{0}{ }^{k+1}-\lambda_{1}{ }^{k+1}\right)}{k ! \mu^{k}(k \mu+v)}+\ldots<0$

which is true. Hence $p_{0} \downarrow$ as $\lambda \uparrow$.

ii) If $\mu_{1}>\mu_{0}$, then

$\frac{p_{0}\left(\lambda, \mu_{1}, v\right)}{\mathrm{p}_{0}\left(\lambda, \mu_{0}, v\right)}>1$

$\Rightarrow \lambda\left(\frac{1}{\mu_{0}}-\frac{1}{\mu_{1}}\right)+\ldots+\frac{\lambda^{k}}{k !}\left(\frac{1}{\mu_{0}^{k}}-\frac{1}{\mu_{1}^{k}}\right)+\frac{\lambda^{k+1} p}{k !}\left\{\frac{1}{\mu_{0}^{k}\left(k \mu_{0}+v\right)}-\frac{1}{\mu_{1}^{k}\left(k \mu_{1}+v\right)}\right\}+\ldots>0$

which is true. Hence $p_{0} \uparrow$ as $\mu \uparrow$

iii) If $v_{1}>v_{0}$, then 
$\frac{p_{0}\left(\lambda, \mu, v_{1}\right)}{\mathrm{p}_{0}\left(\lambda, \mu, v_{0}\right)}>1$

$\Rightarrow \frac{\lambda^{k+1} p}{k ! \mu^{k}}\left\{\frac{1}{\left(k \mu+v_{0}\right)}-\frac{1}{\left(k \mu+v_{1}\right)}\right\}+\frac{\lambda^{k+2} p}{k ! \mu^{k}}\left\{\frac{1}{\left(k \mu+v_{0}\right)\left(k \mu+2 v_{0}\right)}-\frac{1}{\left(k \mu+v_{1}\right)\left(k \mu+2 v_{1}\right)}\right\}+\ldots . .>0$

which is true. Hence $p_{0} \uparrow$ as $v \uparrow$

The following can similarly be shown.

v) $q_{0} \downarrow$ as $\lambda \uparrow$

vi) $q_{0} \uparrow$ as $\mu \uparrow$

vii) $q_{0} \uparrow a s v \uparrow$

The managerial implications of the above results are obvious.

\section{Numerical Example}

To illustrate the use of our results, we apply them to a queuing problem. We quote below an example from Allen (2005, page 352).

'Customers arrive randomly (during the evening hours) at the Kittenhouse, the local house of questionable services, at an average rate of five per hour. Service time is exponential with a mean of 20 minutes per customer. There are two servers on duty.

So many queuing theory students visits the Kittenhouse to collect data for this book that proprietress, Kitty Callay (also known as the Cheshire Cat) make some changes. She trains her kittens to provide more exotic but still exponentially distributed service and add three more servers, for a total of five. Her captivated, customers still complain that the queue is too long. Kitty commissions her most favoured customer Gralre K. Renga to make a study of her establishment. He is to determine the...., the number of servers she should provide so that....the probability that an arriving customers must wait for service will not exceed 0.25 .'

This is a design problem. Here $\lambda=5 / \mathrm{hr}$ and $\mu=3 / \mathrm{hr}$. As required by the owner of the Kittenhouse, we examine the minimum number of servers with different choices of $\mathrm{k}$. Though not explicitly mentioned, it is necessary to assume reneging and balking.

Let us consider a possible markovian reneging rate of $v=0.5 / \mathrm{hr}$. We further assume that balking rate is dependent of state and is 0.1 .

Various performance measures of interest computed are given in the following Table. These measures were arrived at using a FORTRAN 77 program coded by the authors. Different choices of $\mathrm{k}$ were considered. Results relevant with regard to the requirement that the Kittenhouse should provide servers so that the probability that an arriving customers will find all servers busy should be $<0.25$ are presented in the table. (All rates in the table as per hour rates).
Table 1: Table of Performance Measures (with $\lambda=5, \mu=3$, $\nu=0.5$ and $\mathrm{p}=0.9)$

\begin{tabular}{|c|c|c|c|}
\hline \multirow{2}{*}{ Performance Measure } & \multicolumn{3}{|c|}{ Number of servers } \\
\cline { 2 - 4 }$\sum_{n=k+1}^{\infty} p_{n}$ & 0.55616 & 0.25069 & 0.09145 \\
\hline $\begin{array}{c}\lambda^{\mathrm{s}} \text { (i.e. arrival rate of customers } \\
\text { reaching service station) }\end{array}$ & 4.16915 & 4.71970 & 4.91401 \\
\hline Effective mean arrival rate $\left(\lambda^{\mathrm{e}}\right)$ & 4.47006 & 4.80316 & 4.93557 \\
\hline Fraction of time server is idle $\left(\mathrm{p}_{0}\right)$ & 0.16644 & 0.18476 & 0.18822 \\
\hline Average length of queue & 0.60181 & 0.16690 & 0.04312 \\
\hline Average length of system & 1.99152 & 1.74013 & 1.68113 \\
\hline Mean reneging rate & 0.30090 & 0.08345 & 0.02156 \\
\hline $\begin{array}{c}\text { Proportion of customers lost due } \\
\text { to reneging, and balking. }\end{array}$ & 0.16615 & 0.05606 & 0.01719 \\
\hline
\end{tabular}

From the above table it is clear that an ideal choice of $\mathrm{k}$ could be $\mathrm{k}=4$ with

$\sum_{n=k+1}^{\infty} p_{n}=0.09145$. Under the assumption of balking and reneging, it appears that the proprietress need not increase the number of servers to five. Her design requirement would be met with four servers. She may therefore increase the number of servers by two.

\section{Conclusion}

The analysis of a multi server Markovian queuing system with state-dependent balking and Markovian reneging has been presented. Though these concepts were discussed in literature, explicit expressions for $\mathrm{M} / \mathrm{M} / \mathrm{k}$ system are not available. This paper makes a contribution in this direction. Closed form expressions of number of performance measures have been presented. To study the change in the system corresponding to change in system parameters sensitivity analysis has also been presented. A numerical example with design connotations has been presented to demonstrate results derived.

\section{Appendix}

\subsection{Derivation of $P^{\prime}(1)$ under $R \_B O S$.}

Let $\mathrm{P}(\mathrm{s})$ denote the probability generating function, defined by $P(s)=\sum_{n=0}^{\infty} p_{n} s^{n}$

From equation (3.2) we have

$\lambda p_{n-1}+(n+1) \mu p_{n+1}=\lambda p_{n}+n \mu p_{n} ; \quad \mathrm{n}=1,2 \ldots \mathrm{k}-1$.

Multiplying both sides of the equation by $\mathrm{s}^{\mathrm{n}}$ and summing over $n$ 
$\lambda s \sum_{n=1}^{k-1} p_{n-1} s^{n-1}-\lambda \sum_{n=1}^{k-1} p_{n} s^{n}=\sum_{n=1}^{k-1} \mu p_{n} s^{n}-\frac{1}{s} \mu \sum_{n=1}^{k-1}(n+1) p_{n+1} s^{n+1}$

From (3.3) we have

$\lambda p^{n-k} p_{n-1}+\{k \mu+(n-k+1) v\} p_{n+1}=\lambda p^{n-k} p_{n}+\{k \mu+(n-k) v\} p_{n} \quad ; \mathrm{n}=\mathrm{k}, \mathrm{k}+1, \ldots$

Similarly multiplying both sides of the equation by $\mathrm{s}^{\mathrm{n}}$ and summing over $\mathrm{n}$

$\lambda s \sum_{n=k}^{\infty} p^{n-k} p_{n-1} s^{n-1}-\lambda \sum_{n=k}^{\infty} p^{n-k+1} p_{n} s^{n}=\sum_{n=k}^{\infty}\{k \mu+(n-k) v\} p_{n} s^{n}-\frac{1}{s} \sum_{n=k}^{\infty}\{k \mu+(n-k+1) v\} p_{n+1} s^{n+1}$

Adding (8.1.1) and (8.1.2)

$\Rightarrow \lambda s\left[\sum_{n=1}^{k-1} p_{n-1} s^{n-1}+\sum_{n=k}^{\infty} p^{n-k} p_{n-1} s^{n-1}\right]-\lambda\left[\sum_{n=}^{k-1} p_{n} s^{n}+\sum_{n=k}^{\infty} p^{n-k+1} p_{n} s^{n}\right]$

$=\mu s \sum_{n=1}^{k-1} n p_{n} s^{n-1}+\sum_{n=k}^{\infty}\{k \mu+(n-k) v\} p_{n} s^{n}-\frac{1}{s}\left[\mu \sum_{n=1}^{k-1}(n+1) p_{n+1} s^{n+1}+\sum_{n=k}^{\infty}\{k \mu+(n-k+1) v\} p_{n+1} s^{n+1}\right]$

$\Rightarrow \lambda s\left[p_{0} s^{0}+p_{1} s^{1}+\ldots .+p_{k-2} s^{k-2}+p_{k-1} s^{k-1}+\left\{p p_{k} s^{k}+p^{2} p_{k+1} s^{k+1}+\ldots\right\}\right]-\lambda\left\{P(s)-p_{0}-\sum_{n=k}^{\infty} p_{n} s^{n}\right\}-\lambda\left\{p p_{k} s^{k}+p^{2} p_{k+1} s^{k+1}+\ldots\right\}$

$=\mu s\left\{P^{\prime}(s)-\sum_{n=k+1}^{\infty} n p_{n} s^{n-1}\right\}+k \mu\left\{P(s)-\sum_{n=0}^{k} p_{n} s^{n}\right\}+v s\left\{(k+1) p_{k+1} s^{k+1}+\ldots\right\}-v k\left\{p_{k+1} s^{k+1}+\ldots\right\}$

$\frac{1}{s}\left[\mu s\left\{P^{\prime}(s)-p_{1}-\sum_{n=k+1}^{\infty} n p_{n} s^{n-1}\right\}+k \mu\left\{P(s)-\sum_{n=0}^{k} p_{n} s^{n}\right\}+v s\left\{(k+1) p_{k+1} s^{k+1}+\ldots.\right\}-k v\left\{p_{k+1} s^{k+1}+\ldots ..\right\}\right]$

$\Rightarrow \lambda s\left\{P(s)-\sum_{n=k}^{\infty} p_{n} s^{n}\right\}+\left(\lambda s / p^{k-1}\right)\left\{p^{k} p_{k} s^{k}+p^{k+1} p_{k+1} s^{k+1}+\ldots\right\}-\lambda\left\{P(s)-p_{0}-\sum_{n=k}^{\infty} p_{n} s^{n}\right\}-\left(\lambda / p^{k-1}\right)\left\{p^{k} p_{k} s^{k}+p^{k+1} p_{k+1} s^{k+1}+\ldots\right\}$

$=\mu s\left\{P^{\prime}(s)-\sum_{n=k+1}^{\infty} n p_{n} s^{n-1}\right\}+k \mu\left\{P(s)-\sum_{n=0}^{k} p_{n} s^{n}\right\}+v\left\{\left\{P^{\prime}(s)-\sum_{n=1}^{k} n p_{n} s^{n-1}\right\}-k v\left\{P(s)-\sum_{n=0}^{k} p_{n} s^{n}\right\}\right.$

$-\mu\left\{P^{\prime}(s)-p_{1}-\sum_{n=k+1}^{\infty} n p_{n} s^{n-1}\right\}-\frac{k \mu}{s}\left\{P(s)-\sum_{n=0}^{k} p_{n} s^{n}\right\}-v\left\{P^{\prime}(s)-\sum_{n=1}^{k} n p_{n} s^{n-1}\right\}+\frac{k v}{s}\left\{P(s)-\sum_{n=0}^{k} p_{n} s^{n}\right\}$

$\Rightarrow \lambda s\left\{P(s)-\sum_{n=k}^{\infty} p_{n} s^{n}\right\}+\left(\lambda s / p^{k-1}\right)\left\{P(p s)-\sum_{n=0}^{k-1} p_{n}(p s)^{n}\right\}-\lambda\left\{P(s)-p_{0}-\sum_{n=k}^{\infty} p_{n} s^{n}\right\}-\left(\lambda / p^{k-1}\right)\left\{P(p s)-\sum_{n=0}^{k-1} p_{n}(p s)^{n}\right\}$

$=\mu s\left\{P^{\prime}(s)-\sum_{n=k+1}^{\infty} n p_{n} s^{n-1}\right\}+k \mu\left\{P(s)-\sum_{n=0}^{k} p_{n} s^{n}\right\}+v s\left\{P^{\prime}(s)-\sum_{n=1}^{k} n p_{n} s^{n-1}\right\}-k v\left\{P(s)-\sum_{n=0}^{k} p_{n} s^{n}\right\}$

$-\mu\left\{P^{\prime}(s)-p_{1}-\sum_{n=k+1}^{\infty} n p_{n} s^{n-1}\right\}-\frac{k \mu}{s}\left\{P(s)-\sum_{n=0}^{k} p_{n} s^{n}\right\}-v\left\{P^{\prime}(s)-\sum_{n=1}^{k} n p_{n} s^{n-1}\right\}+\frac{k v}{s}\left\{P(s)-\sum_{n=0}^{k} p_{n} s^{n}\right\}$

$\Rightarrow \lambda s P(s)-\lambda s \sum_{n=k}^{\infty} p_{n} s^{n}+\lambda s P(p s) / p^{k-1}-\left\{\lambda s \sum_{n=0}^{k-1} p_{n}(p s)^{n}\right\} / p^{k-1}-\lambda P(s)+\lambda p_{0}+\lambda \sum_{n=k}^{\infty} p_{n} s^{n}-\lambda P(p s) / p^{k-1}+\left\{\lambda \sum_{n=0}^{k-1} p_{n}(p s)^{n}\right\} / p^{k-1}$

$=\mu s P^{\prime}(s)-\mu s \sum_{n=k+1}^{\infty} n p_{n} s^{n-1}+k \mu P(s)-k \mu \sum_{n=0}^{k} p_{n} s^{n}+v s P^{\prime}(s)-v s \sum_{n=1}^{k} n p_{n} s^{n-1}-k v P(s)+k v \sum_{n=0}^{k} p_{n} s^{n}$

$-\mu P^{\prime}(s)+\mu \frac{\lambda}{\mu} p_{0}+\mu \sum_{n=k+1}^{\infty} n p_{n} s^{n-1}-\frac{k \mu}{s} P(s)+\frac{k \mu}{s} \sum_{n=0}^{k} p_{n} s^{n}-v P^{\prime}(s)+v \sum_{n=1}^{k} n p_{n} s^{n-1}+\frac{k v}{s} P(s)-\frac{k v}{s} \sum_{n=0}^{k} p_{n} s^{n}$

$\Rightarrow P^{\prime}(s)(\mu+v)=\lambda P(s)-\lambda \sum_{n=k}^{\infty} p_{n} s^{n}+\lambda P(p s) / p^{k-1}-\left\{\lambda \sum_{n=0}^{k-1} p_{n}(p s)^{n}\right\} / p^{k-1}+\mu \sum_{n=k+1}^{\infty} n p_{n} s^{n-1}-\frac{k \mu}{s} P(s)+\frac{k \mu}{s} \sum_{n=0}^{k} p_{n} s^{n}$

$+v \sum_{\mathrm{n}=1}^{\mathrm{k}} n p_{n} s^{n-1}+\frac{k v}{s} P(s)-\frac{k v}{s} \sum_{n=0}^{k} p_{n} s^{n}$ 


\section{International Journal of Science and Research (IJSR) \\ ISSN (Online): 2319-7064}

Now

$$
\begin{aligned}
& \lim P^{\prime}(s)=\lim \frac{1}{(\mu+v)}\left[\begin{array}{l}
\lambda P(s)-\lambda \sum_{n=k}^{\infty} p_{n} s^{n}+\lambda P(p s) / p^{k-1}-\left\{\lambda \sum_{n=0}^{k-1} p_{n}(p s)^{n} / p^{k-1}+\mu \sum_{n=k+1}^{\infty} n p_{n} s^{n-1}-\frac{k \mu}{s} P(s)+\frac{k \mu}{s} \sum_{n=0}^{k} p_{n} s^{n}\right. \\
+v \sum_{n=1}^{k} n p_{n} s^{n-1}+\frac{k v}{s} P(s)-\frac{k v}{s} \sum_{n=0}^{k} p_{n} s^{n}
\end{array}\right] \\
& s \rightarrow 1-\quad s \rightarrow 1- \\
& \Rightarrow P^{\prime}(1)=\frac{1}{(\mu+v)}\left[\lambda\left(1-\sum_{n=k}^{\infty} p_{n}\right)+\left(\lambda / p^{k-1}\right)\left\{P(p)-\sum_{n=0}^{k-1} p_{n} p^{n}\right\}+\mu\left\{P^{\prime}(1)-\sum_{n=1}^{k} n p_{n}\right\}-k \mu\left(1-\sum_{n=0}^{k} p_{n}\right)+v \sum_{n=1}^{k} n p_{n}+k v\left(1-\sum_{n=0}^{k} p_{n}\right)\right] \\
& \Rightarrow P^{\prime}(1)=(1 / v)\left[\lambda\left(1-\sum_{n=k}^{\infty} p_{n}\right)+\left(\lambda / p^{k-1}\right)\left\{P(p)-\sum_{n=0}^{k-1} p_{n} p^{n}\right\}-(\mu-v) \sum_{n=1}^{k} n p_{n}-k(\mu-v)\left(1-\sum_{n=0}^{k} p_{n}\right)\right]
\end{aligned}
$$

Here $P(p)=\sum_{n=0}^{\infty} p_{n}(\lambda, \mu, v) p^{n}$ where the symbol $p_{n}(p \lambda, \mu, v)$ is as described in section 5. We use $p_{n}$ and $p_{n}(\lambda, \mu, v)$ interchangeably. However should any of the parameters $\lambda, \mu, v$ change, it is explicitly stated. To obtain a closed form expression for $\mathrm{P}(\mathrm{p})$, let us for the time being, $p_{n}(p \lambda, \mu, v)=\left\{(p \lambda)^{n} / n ! \mu^{n}\right\} p_{0}(p \lambda, \mu, v) ; \mathrm{n}=1,2 \ldots \mathrm{k}$, $p_{n}(p \lambda, \mu, v)=\left[(p \lambda)^{n} p^{\{(n-k)(n-k+1)\} / 2} / k ! \mu^{k} \prod_{r=k+1}^{n}\{\mu+(r-k) v\}\right] p_{0}(p \lambda, \mu, v) ; \mathrm{n}=\mathrm{k}+1, \ldots$

where

$$
p_{0}(p \lambda, \mu, v)=\left[\sum_{n=0}^{k}(p \lambda)^{n} / n ! \mu^{n}+\sum_{n=k+1}^{\infty}(p \lambda)^{n} p^{\{(n-k)(n-k+1)\} / 2} / k ! \mu^{k} \prod_{r=k+1}^{n}\{\mu+(r-k) v\}\right]^{-1} .
$$

Let $P(S ; p \lambda, \mu, v)$ denotes the probability generating function of this new queuing system so that $P(S ; p \lambda, \mu, v)=\sum_{n=0}^{\infty} p_{n}(p \lambda, \mu, v) s^{n}$.

Now

$$
\begin{aligned}
& P(p)=\sum_{n=0}^{\infty} p_{n}(\lambda, \mu, v) p^{n} \\
& =p_{0}+\sum_{n=1}^{k}(p \lambda)^{n} p_{0} / n ! \mu^{n}+\sum_{n=k+1}^{\infty}\left[(p \lambda)^{n} p^{\{(n-k)(n-k+1)\} / 2} / k ! \mu^{k} \prod_{r=k+1}^{n}\{\mu+(r-k) v\}\right] p_{0} \\
& \Rightarrow\left[\left(P(p)-p_{0}\right) / p_{0}\right]=\sum_{n=1}^{k}(p \lambda)^{n} / n ! \mu^{n}+\sum_{n=k+1}^{\infty}\left[(p \lambda)^{n} p^{\{(n-k)(n-k+1)\} / 2} / k ! \mu^{k} \prod_{r=k+1}^{n}\{\mu+(r-k) v\}\right] .
\end{aligned}
$$




$$
\begin{aligned}
& P(1 ; p \lambda, \mu, v)=p_{0}(p \lambda, \mu, v)+\sum_{n=1}^{\infty} p_{n}(p \lambda, \mu, v) \\
& \Rightarrow 1=p_{0}(p \lambda, \mu, v)+\sum_{n=1}^{k}(p \lambda)^{n} / n ! \mu^{n}+\sum_{n=k+1}^{\infty}\left[(p \lambda)^{n} p^{\{(n-k)(n-k+1)\}} / k ! \mu^{k} \prod_{r=k+1}^{n}\{\mu+(r-k) v\}\right] p_{0}(p \lambda, \mu, v) \operatorname{using}(8.1 .4)_{\mathrm{A}} \\
& \Rightarrow 1=p_{0}(p \lambda, \mu, v)+\left\{\left(P(p)-p_{0}\right) / p_{0}\right\} p_{0}(\mathrm{p} \lambda, \mu, v) \\
& \Rightarrow P(p)=p_{0} / p_{0}(p \lambda, \mu, v)
\end{aligned}
$$$$
\text { gain let } K_{R_{-} B O S}(p \lambda, \mu, v)=\sum_{n=k}^{\infty} p_{n}(p \lambda, \mu, v)
$$$$
=\sum_{n=k}^{\infty}\left[(p \lambda)^{n} p^{\{(n-k)(n-k+1)\} / 2} / k ! \mu^{k} \prod_{r=k+1}^{n}\{\mu+(r-k) v\}\right] p_{0}(p \lambda, \mu, v)
$$$$
=\sum_{n=k}^{\infty} p_{n} p^{n}\left\{p_{0}(p \lambda, \mu, v) / p_{0}\right\}
$$

Therefore,

$\sum_{n=k}^{\infty} p_{n} p^{n}=p_{0} K_{R_{-} B O S}(p \lambda, \mu, v) / p_{0}(p \lambda, \mu, v)$.

Using these in (8.1.3) we obtain

$P^{\prime}(1)=(1 / v)\left[\lambda\left(1-K_{R_{-} B O S}\right)+\left\{\lambda p_{0} K_{R_{-} B O S}(p \lambda, \mu, v) / p^{k-1} p_{0}(p \lambda, \mu, v)\right\}-(\mu-v) \sum_{n=1}^{k} n p_{n}-k(\mu-v)\left(K_{R_{-} B O S}-p_{k}\right)\right]$.

where $\mathrm{p}_{0}(\mathrm{p} \lambda, \mu, v)$ is given in (8.1.5).

\subsection{Derivation of $Q^{\prime}(1)$ under $R_{-}$EOS}

From equation (3.9) we have,

$\lambda q_{n-1}+(n+1)(\mu+v) q_{n+!}=\lambda q_{n}+n(\mu+v) q_{n} ; \quad \mathrm{n}=1,2, \ldots \mathrm{k}-1$

Multiplying both sides of this equation by $\mathrm{s}^{\mathrm{n}}$ and summing over $\mathrm{n}$ from we get

$$
\lambda s \sum_{n=1}^{k-1} q_{n-1} s^{n-1}-\lambda \sum_{n=1}^{k-1} q_{n} s^{n}=(\mu+v) \sum_{n=1}^{k-1} n q_{n} s^{n}-\frac{1}{s}(\mu+v) \sum_{n=1}^{k-1}(n+1) q_{n+1} s^{n+1}
$$

From equation (3.10)

$\lambda p^{n-k} q_{n-1}+\{k \mu+(n+1) v\} q_{n+1}=\lambda p^{n-k+1} q_{n}+\{k \mu+n v\} q_{n}$ $; \mathrm{n}=\mathrm{k}+1, \mathrm{k}+2, \ldots$

Multiplying both sides of this equation by $\mathrm{s}^{\mathrm{n}}$ and summing over $\mathrm{n}$ from we get

$$
\lambda s \sum_{n=k+1}^{\infty} p^{n-k} q_{n-1} s^{n-1}-\lambda \sum_{n=k+1}^{\infty} p^{n-k+1} q_{n} s^{n}=\sum_{n=k+1}^{\infty}\{k \mu+n v\} q_{n} s^{n}-\frac{1}{s} \sum_{n=k+1}^{\infty}\{k \mu+(n+1) v\} q_{n+1} s^{n+1} \text {. }
$$

Adding (8.2.1) and (8.2.2) and proceeding in a manner similar to section (8.1), we obtain,

$$
\Rightarrow Q^{\prime}(1)=\frac{1}{v}\left[\lambda\left(1-K_{R_{-} E O S}\right)+\left\{\lambda q_{0} K_{R_{-} E O S}(p \lambda, \mu, v)\right\} / p^{k-1} q_{0}(p \lambda, \mu, v)-\mu \sum_{n=1}^{k} n q_{n}-k \mu\left(K_{R_{-} E O S}-q_{k}\right)\right] \text {. }
$$

$$
\begin{aligned}
& \text { where } q_{0}(p \lambda, \mu, v)=\left[\sum_{n=0}^{k}(p \lambda)^{n} / n ! \mu^{n}+\sum_{n=k+1}^{\infty}(p \lambda)^{n} p^{\{(n-k)(n-k+1)\} / 2} / k ! \mu^{k} \prod_{r=k+1}^{n}\{\mu+r v\}\right]^{-1} \\
& \text { and } K_{R_{-} B O S}(p \lambda, \mu, v)=\sum_{n=k}^{\infty} q_{n}(p \lambda, \mu, v)
\end{aligned}
$$

\section{Volume 6 Issue 1, January 2017




\section{International Journal of Science and Research (IJSR) \\ ISSN (Online): 2319-7064}

Index Copernicus Value (2015): 78.96 | Impact Factor (2015): 6.391

\section{References}

[1] Allen, A. (2005). Probability, Statistics and Queuing Theory with Computer Science Application, Second Edition, Academic Press.

[2] Altman, E. and Yechiali, U. (2008). Infinite- Server Queues with System's Additional Tasks and Impatient Customers. Engineering and Informational Science., 22, pp: 477-493.

[3] Bae, J., Kim, S. and Lee, E.Y. (2001). The virtual waiting time of the $\mathrm{M} / \mathrm{G} / 1$ Queue with Impatient Customers. Queuing System, 38, pp: 485-494.

[4] Baccelli,F. ,Boyer, P. and Hebuterne, G. (1984). Single Server queues with Impatients Customers, Adv. Appl. Prob., 16,887-905.

[5] Barrer, D.Y. (1958). Queuing with impatient customers and ordered Service. Operations Research, vol.6, pp: 650-656.

[6] Barrer, D.Y. (1957). Queuing with impatient customers and indifferent clerks. Operations Research, vol. 5, pp: 644-649.

[7] Boots, N.K. and Tijms, H. (1999). A multi server Queuing System with Impatient Customers. Management Science, 45, 3, pp: 444-448.

[8] Boxma, O. J., Kella, O., Perry, D. and Prabhu, B.J. (2008). Proc. of IWAP 2008, July 7-10, 2008, compiegne, France.

[9] Brandt, A. and Brandt, M. (1998). On a Two- Queue Priority System with Impatience and its Application to a Call Centre. Konrad-Zuse-Zentrum fur Informationstechnik Berlin. pp: 1-28.

[10] Choi, B. D., Kim, B. and Chung, J. (2001) . M/M/1 Queue with Impatient Customers of Higher Priority. Queuing System, 38, 1, pp: 49-66.

[11] Choi. B. D., Kim, B. and Zhu, D. (2004). MAP/M/C Queue with Constant Impatients Times. Mathematics of Operations Research, 29, 2, pp: 309-325.

[12] Choudhury, A. (2008). Impatience in Single Server Queuing Model. American Journal of Mathematical and Management Sciences, Vol. 28, pp: 177-211.

[13] Choudhury, A (2009). A few words on Reneging in $\mathrm{M} / \mathrm{M} / 1 / \mathrm{K}$ queues. Contributions to Applied and Mathematical Statistics, 4, pp: 58-64.

[14] El-Paoumy, M. S. (2008). On Poisson Bulk Arrival Queue: $\mathrm{M}^{\mathrm{X}} / \mathrm{M} / 2 / \mathrm{N}$ with Balking, Reneging and Heterogeneous Servers. Applied Mathematical Sciences, Vol. 2, No. 24, pp: 1169-1175.

[15]El-Paoumy, M.S. and Ismail, M. M. (2009). On a Truncated Erlang Queuing System with Bulk Arrivals, Balking and Reneging . Applied Mathematical Sciences, Vol. 3, No. 23, pp: 1103-1113.

[16]El-Sherbiny, A. A. (2008). The Non-Truncated Bulk Arrival Queue $\mathrm{M}^{\mathrm{X}} / \mathrm{M} / 1$ with reneging, Balking, StateDependent and an Additional Server for Longer Queues. Applied Mathematical Sciences, Vol. 2, No. 15, pp: 747-752.

[17] Gavish, B. and Schweitzer, P.J. (1977). The Markovian Queue with Bounded Waiting Time. Management Science, 23, 12, pp: 1349-1357.

[18] Ghoshal, A. (1963). Queues with Finite Waiting Time. Operations Research, 11, pp: 919-921.
[19] Hagighi, A. Montazer, Medhi, J. and Mohanty, S. G. (1986). On a multi server markovian queuing system with Balking and Reneging. computer and Operational Research. Vol.13, No. 4, pp: 421-425.

[20] Haight, F.A. (1957). Queuing with Balking. Biometrika, 44. , pp: 360-369.

[21]Haight, F.A. (1959). Queuing with Reneging. Biometrika, 2. , pp: 186-197.

[22] Ke, J. C. and Wang, K-H. (1999). Cost analysis of the $\mathrm{M} / \mathrm{M} / \mathrm{R}$ machine repair problem with balking, reneging and server breakdowns. Journal of the Operational Research Society, 50, pp: 275-282.

[23]Kok, A.G.D and Tijms, H. (1985). A Queuing System with Impatient customers. J. Appl. Prob, 22, pp: 688696.

[24] Liu, L. and Kulkarni, V.G. (2008). Busy Period analysis for $\mathrm{M} / \mathrm{PH} / 1$ queues with workload dependent Balking. Queuing System, 59, pp: 37-51.

[25]Liu, L, Kashyap, B. R. K. and Templeton, J. G. C. (1987). The service system $\mathrm{M} / \mathrm{M}^{\mathrm{R}} / \infty$ with impatient customers. Queuing System 2, pp: 363-372.

[26] Martin, M. and Artalejo, J. R. (1995). Analysis of an M/G/1 Queue with two types of Impatient units. Adv. Appl. Prob., 27, pp: 840-861.

[27] Medhi, J. (2003). Stochastic model in Queuing theory, Second Edition, Academic Press.

[28] Ravindran, A, Phillips, Don T. and Solberg, J. J. (1987). Operations Research, Principles and practice, Second Edition, John Wiley and Sons. ( New York).

[29] Shawky, A. I. (1997). The single server machine interference model with balking, reneging and an additional server for longer queues. Microelectron Reliability, Vol. 37, pp: 355-357.

[30] Singh, C.J., Kumar, B. and Jain, M. (2007) .Single server interdependent queuing model with controllable arrival rates and reneging. Pakistan J. Stat. 23, No. 2, pp: 171-178.

[31]Zhang, Y., Yue, d. and Yue, W. (2005). Analysis of an $\mathrm{M} / \mathrm{M} / 1 / \mathrm{N}$ queue with balking, reneging and server vacations. Proc. Vth. Inter. Symp, pp: 37-47. 